18 | 2014

NOVECENTO... E DINTORNI

Da Torino a Parigi: Laura Malvano storica e critica d'arte

\title{
Laura par Nella
}

Laura di Nella

Laura by Nella

Patrick Mayoux

\section{OpenEdition}

Journals

Édition électronique

URL : https://journals.openedition.org/cei/1579

DOI : $10.4000 /$ cei. 1579

ISSN : 2260-779X

Éditeur

UGA Éditions/Université Grenoble Alpes

Édition imprimée

Date de publication : 30 mars 2014

Pagination : 73-83

ISBN : 978-2-84310-268-4

ISSN : $1770-9571$

\section{Référence électronique}

Patrick Mayoux, «Laura par Nella », Cahiers d'études italiennes [En ligne], 18 | 2014, mis en ligne le 30 septembre 2015, consulté le 21 septembre 2021. URL : http://journals.openedition.org/cei/1579 ; DOI : https://doi.org/10.4000/cei. 1579 


\title{
LAURA PAR NELLA
}

\author{
Patrick Mayoux
}

Pour tenter, en paroles, un portrait de Laura Malvano, on voudrait évoquer ici quelques-uns des tableaux d'elle peints par sa mère Nella, si émouvante par sa peinture, si impressionnante par l'intensité de sa vie écourtée. Cette femme a poussé l'attention envers ses enfants jusqu'à les songer en les portant sur la toile par le dur travail de peinture. Non pas seulement songer à eux, et non pas seulement retenir, préserver cet instant de vie, ce moment, cette période que voici, ils vont disparaître, que voilà, ils ont disparu. Mais parvenir à songer ses enfants, pour nous ici ce sera Laura, par l'acte de peindre; songer Laura en donnant son image, faire ainsi transparaître sa trame imaginaire, et préfigurer les possibles de sa vie. Ces tableaux nous paraissent offrir une seconde naissance au modèle qui fut peint. Re-naissance requise pour chacun, et qui fut dans ce cas, croyons-nous, ressentie comme telle par Laura. Acte d'amour, ou de charité, selon le parler de Nella Marchesini.

En observant quelques tableaux, tentons de faire sentir comment ce don d'images venu de sa mère a pu indiquer à Laura la voie de sa propre vie.

La séquence des dix tableaux examinés ci-après n'est pas chronologique. Elle n'est pas non plus une anthologie des tableaux que nous préférons, ou que nous estimerions les plus importants. Elle vise seulement à soumettre cette idée d'une peinture "augurale" à une lecture qu'on a voulue précise, y compris, ou surtout, sur des détails, d'œuvres qu'on a crues pertinentes pour cette tentative.

\section{Autoritratto con le figlie}

Ce tableau peine à contenir les trois personnages, à les empêcher de quitter la toile, de venir à nous — tant leur présence est forte. La mère accoudée, 
pensive, et à sa droite juste derrière elle les deux filles se découpant l'une derrière l'autre. Elles sont tendues l'une et l'autre par l'action en elles d'un sentiment intense. La première est dans l'effarement, Laura juste à l'arrière paraît la seconder, selon une empathie qui la conduit à la tristesse. Cette œuvre est datée d'environ 1945, à cette date Laura est déjà adolescente, cependant ses proportions, son visage et le maintien choisis sur le tableau la dépeignent encore enfant, de même que sa sœur. Son visage présente en lumière la moitié gauche, l'autre est en demi-teinte, cependant la bouche apparaît tout entière. De même pour la mère, dont la moitié gauche du visage est prise par l'ombre, alors que sa bouche retient elle aussi la lumière. Chez Nella Marchesini, il y a une très grande sûreté dans le modelé des postures. Dressées derrière la mère assise, les enfants semblent ici à elles seules constituer un chœur de témoins.

De quel triste fait iraient-elles témoigner, devant cette question évitons les hypothèses, d'autant que la portée allégorique des tableaux de Marchesini transcende circonstances et faits. Il est frappant de voir que l'expression dramatique est dévolue aux petites, tandis que la mère paraît se contenir dans le souci, grave souci qui n'est d'ailleurs pas visible pour ses filles se tenant à l'arrière. Mater meditativa, dum deplorant filiae. Ce tableau prend un risque figuratif, dans la peinture du bras droit de ce personnage maternel : d'abord, toute la longueur du bras est prise par un ton rouge sombre, plus foncé que l'ombre qui mange la moitié du visage, plus que l'ombre couvrant le bras gauche sur lequel s'appuie la tête lasse - comme si la couleur du sang paraissait directement et massivement sous une peau transparente. Ensuite, il y a un contraste marqué entre le volume affiné, fuselé de l'épaule jusqu'au coude, et la grosseur de l'avantbras; contraste encore accentué par la main droite qui repose de chant sur la table, au tout premier plan du tableau. Cette main paraît à son tour disproportionnée par rapport à l'avant-bras, lui-même si fort par rapport à la première partie du bras. Nous insistons sur le signe figuré par la main, car on le verra reparaître dans plusieurs tableaux représentant Laura seule. Ici, devant la mère et ses filles, on se dit que la mère est à l'arrêt, se sent au bout de ce qu'elle peut, malgré sa volonté n'aperçoit plus ses ressources, et que ses filles sentent cela, et pressentent une adversité aux mille têtes.

\section{Laura che fa i compiti}

C'est Laura enfant, elle s'applique à ses devoirs scolaires. Ici à nouveau, le visage est bipartite, à valeurs inversées cette fois : lumière à droite, ombre à 
gauche. La main gauche de l'écolière est à plat sur le cahier, sa main droite, à son tour bipartite ombre et lumière, est appliquée à écrire. Le buste et les bras, vêtus de bleu marine, apparaissent requis par la tâche. Cependant les paupières abaissées suggèrent davantage qu'un regard studieux, et qui ne se laisse pas voir. Au-delà, toute l'expression du visage suggère un rêve de sérieux, une enfant qui essaie son imagination du côté du sérieux. Une imagination en marche, pour s'approprier le sérieux et la volonté. À l'avant-plan, un encrier gris-bleuté, qui a des allures de gramophone, veille sur l'écriture de l'enfant. À nous, il peut suggérer le grand-père de Laura (elle n'a pu voir l'homme, disparu avant sa naissance, mais bien sentir son prestige immense aux yeux de Nella), le «babbo sognatore», le vieil homme au rêve. Celui qui avait son rêve chevillé à sa vie, élevant ses enfants selon ce rêve (comme Laura elle-même, dans son profond écrit "Salvare i giorni della vita dalla dimenticanza ${ }^{ }$", citant Nella, le signalera fortement), et laissant ainsi, notamment, la vocation de peindre s'emparer de Nella. Donc, cet encrier lui aussi est bipartite, partagé comme le visage de Laura. Si l'objet qui pourvoit l'encre est bien un intersigne, rapportons-le au grand-père qui a "aimé le savoir plus que la beauté ${ }^{»}$. Peut-être la Laura qui grandira après ce tableau aura-t-elle aimé, à parts égales quant à elle, beauté, lumière, et savoir.

\section{Laura con la rosa}

Voici Laura sans doute un peu plus âgée qu'au tableau précédent. À part le blanc de la fleur, auquel répond le blanc de la blouse de la petite fille, ce sont des tons plus sourds qui dominent ce tableau-ci. La rose blanche sur la gauche est verticale dans son vase, la petite fille aussi est debout, sans ombre cette fois quoique dans une lumière assourdie, les paupières abaissées comme au tableau des devoirs, mais sous un autre signe, cette fois. Car la rose est peinte assez pimpante, et même narquoise. Or il est difficile d'être comme une fleur, étant petite fille. S'il est impossible de l'être toujours, il est déjà difficile de l'être souvent. Et peut-être la petite fille ici présente sur le tableau s'y refuse-t-elle. Et peut-être a-t-elle pour cela des motifs bien forts. À la voir ici, elle n'est pas mignonne, ni pimpante.

I. L. Malvano, "Salvare i giorni della vita dalla dimenticanza”: Pittura e scrittura nell'opera di Nella Marchesini Malvano ", dans Nella Marchesini, catalogue de l'exposition à la Galleria del Ponte, Turin, 9 février3I mars 2006, p. 7-32.

2. Ibid., p. 13 . 
Quelque chose la chiffonne, sans doute, ou même la chagrine. Sa main est posée, peut-être sur un carnet, ou un petit livre, et cette main est assez brute, peinte en tons sombres qui la rapprochent de la main de la mère, sur notre tableau initial. La main de cette petite fille ne paraît pas être une main de petite fille, elle paraît être déjà passée par des tribulations, et devoir en affronter de nouvelles. Ce tableau confronte la petite Laura à une rose «sans pourquoi». Mais sur ce visage incliné, si les paupières sont abaissées sur un songe, c'est un songe contrariant, peut-être alarmant.

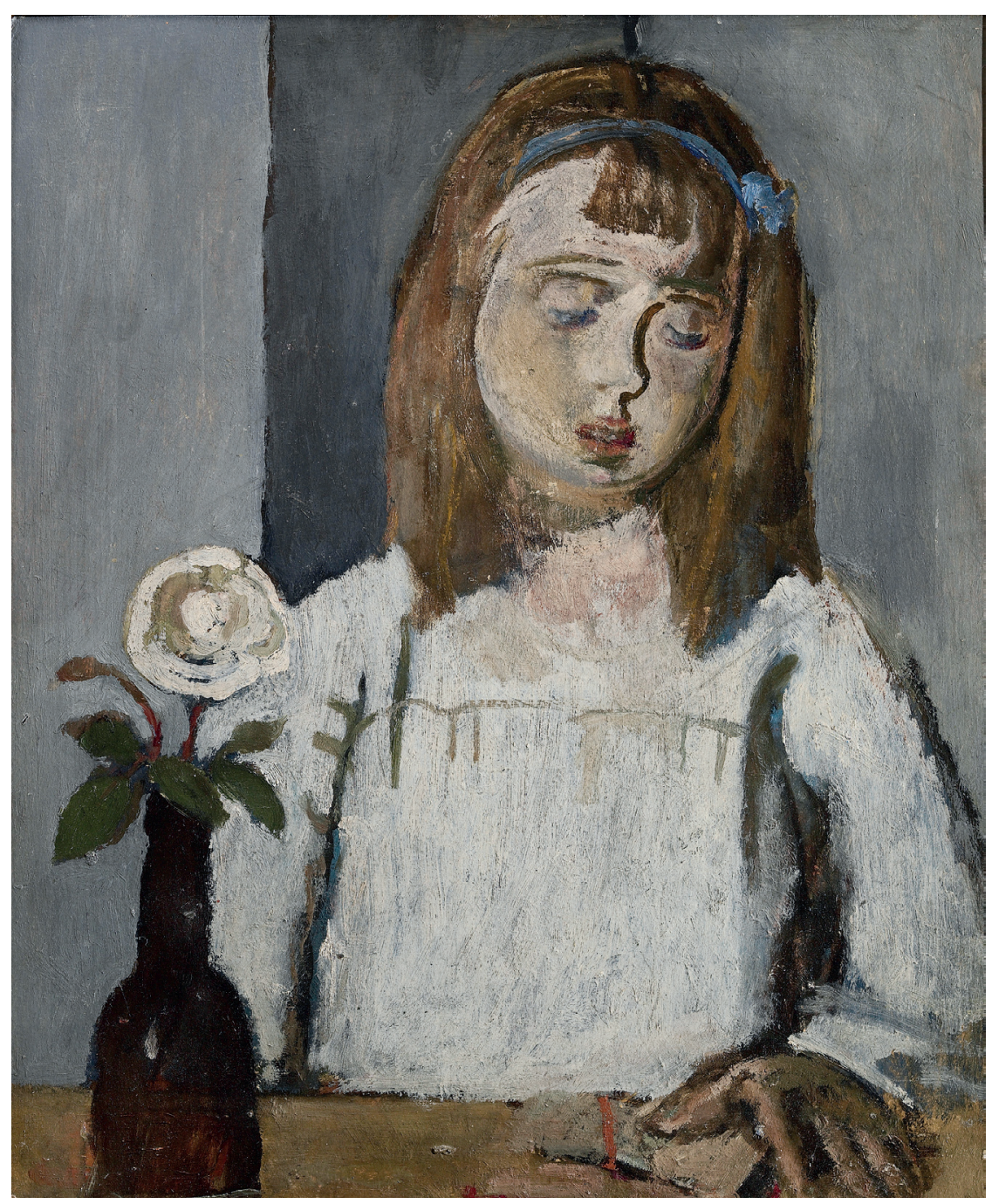

Laura con la rosa. 


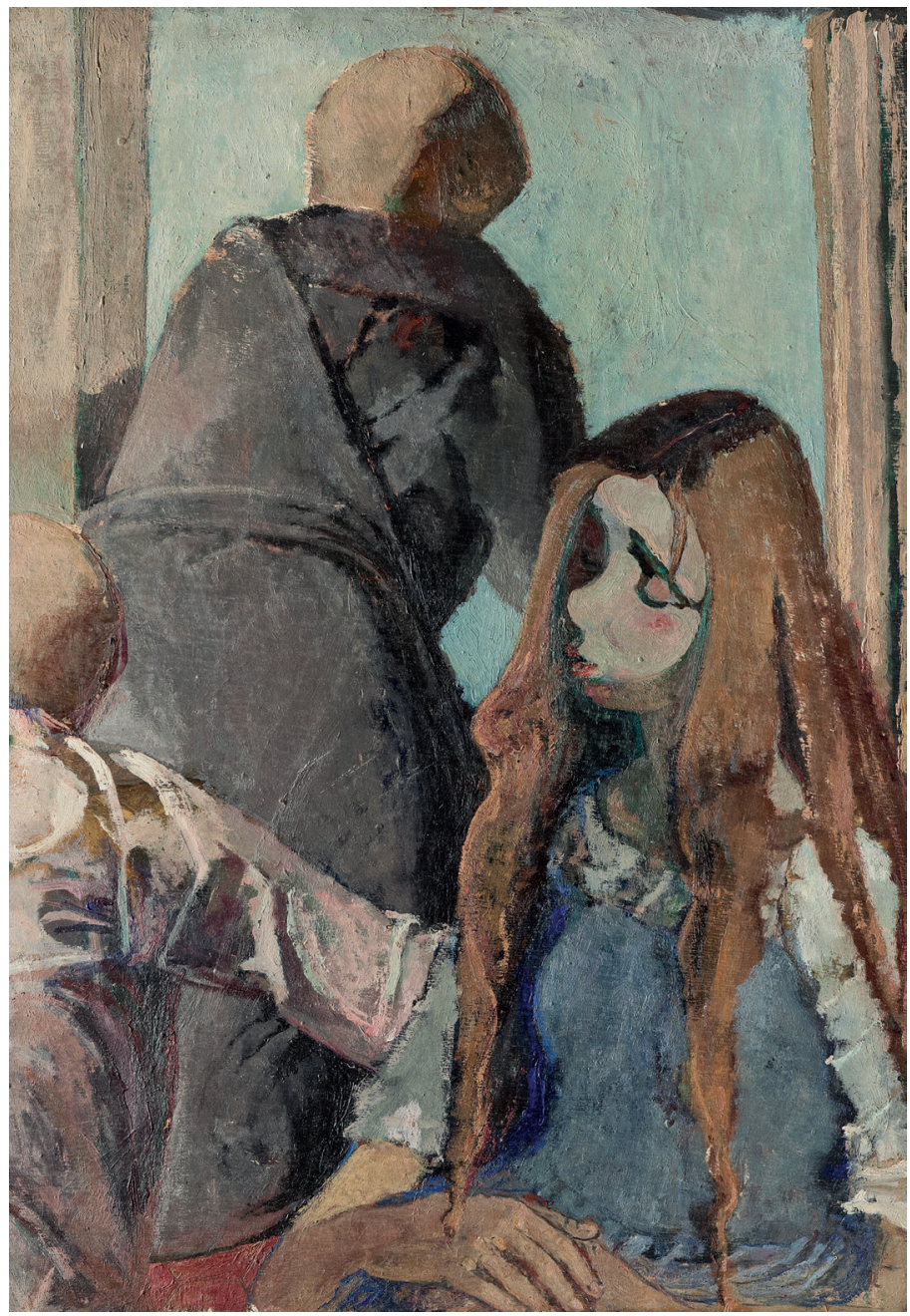

Laura e i manichini.

\section{Laura e i manichini}

Ici la peinture de Marchesini touche à l'étrangeté, et à vrai dire une étrangeté plutôt inquiétante. Deux personnages escaladant et peints de dos occupent une large moitié du tableau, donnant une forte impression d'intrusion bien qu'ils soient sur le point de s'en aller, en traversant le fond, qui paraît plutôt un mur qu'une fenêtre. Une Laura de trois quarts est assise sur la droite, elle est presque méconnaissable. À nouveau, elle a les paupières abaissées, mais surtout, encore plus qu'au tableau à la rose, la voici 
peinte sans nulle intention de joliesse. Son visage aux yeux clos n'est pas inquiet, mais avec ses angles accusés il paraît taillé dans du bois. La chevelure aussi est étrange, partagée en trois ou quatre épaisses longues mèches tombantes qui paraissent des écheveaux de laine. Cette figure pourrait être celle d'une grande marionnette, aux dimensions des deux escaladeurs. Qui sont des mannequins, le titre du tableau nous l'assure, sans dissiper notre inquiétude devant ces êtres animés d'un mouvement d'énergumènes. Émanations que l'on verrait chez Ensor, ou chez De Chirico. Quant à la Laura de ce tableau, ce pourrait être plutôt son double onirique, dans des rêves mal famés, ou encore une poupée qu'elle aurait elle-même taillée à sa ressemblance caricaturale. Mais si c'est une jeune fille, elle paraît déplorer quelque chose. Peut-être pas le départ des mannequins, mais plutôt la propension à nourrir de pareilles visions. Notre intuition, quant à la peinture des personnes chez Nella Marchesini, c'est qu'elle tendait à peindre des prémonitions, avec la visée de prémunir ses modèles contre l'adversité, ou leurs propres démons, en songeant à leur plus haute destinée. Il n'est pas impossible que Laura ait eu des hantises comme celles que ce tableau évoque, il est concevable qu'un tel tableau, sans complaisance, sans euphémisme, l'ait aidée à les contenir. Sa main, à l'avant-plan selon la coutume de Nella, se dessine dans l'ombre, mais n'est pas trop loin d'une main sereine.

\section{Ritratto della figlia Laura}

Voici maintenant l'un des tableaux où Laura se montre paisible. Elle est assise sur une banquette, on imagine un entre-deux, le repos d'un moment. Contours, postures des bras, tout est très fin, délié, et sans apprêt. La longueur des doigts incurvés ou repliés suggère, mais sans la tension du tableau à la rose, que grandir est aussi un problème. Alors que le visage est comme nimbé par son enfance, c'est la dissymétrie qui donne l'animation, qui indique peut-être l'entrechoc des aspirations dont l'enfant se repose dans le moment que retient cette peinture. Dissymétrie légère, des postures des épaules, des bras, des jambes, correspondant à l'inclinaison à peine marquée de l'enfant sur sa droite. Abandon des deux jambes nettement disjointes - et cet abandon insoucieux, au moment d'un repos vacant ou songeur, est d'une enfant de la campagne, dont les jambes courent, marchent ou reposent sur le sol, sans inquiétude envers le regard des autres. Quant à son visage, le voici une fois de plus paupières abaissées, ce qui se laisse aisément comprendre ici comme une interruption de la 
vie active diurne. Mais il est remarquable que sur les quelque quarante tableaux de Nella où paraît Laura, seule ou avec d'autres, environ les deux tiers la montrent les yeux clos. Pour essayer de comprendre ce fait, il faut une étude affinée, à partir de la vision des tableaux eux-mêmes, non de reproductions, nous ne pouvons ici qu'en indiquer la nécessité.

\section{Laura sulla poltrona verde}

Regardons ce tableau qui fait voir Laura les yeux grands ouverts! Oui, mais leur couleur reste estompée, la peinture a opté pour des tons mats, comme ceux cultivés par Braque. Ce visage ouvert, et ce corps déployé paraissent animés par une grande aspiration, un bon vouloir sans borne. Pourtant les bras sont croisés, mais ils se croisent librement, comme pour prendre un élan. Ici à nouveau, il y a une hyperbole sur les mains, leur ampleur est soulignée; la droite, la plus visible, pourrait recouvrir le visage entier et le haut du crâne! Mais si l'enfant a bien grandi, sur le front de la jeune fille le peintre relève un signe d'enfance : ces mèches un peu larges, esquisses de frange qui reviennent sur d'autres tableaux, et qui ont toujours subsisté, nous semble-t-il, chez Laura Malvano, quand sa chevelure envoyait sur son front de petites passerelles.

\section{Laura col gilé bordeaux}

Maintenant voici Laura les yeux très grands ouverts, presque écarquillés, la prunelle bleu-gris ne peut que fixer, laissant paraître tout le pourtour blanc de l'iris. Ces yeux expriment une tension, au bord de l'effroi. À nouveau ici, quelque chose paraît hors de proportion avec la jeune fille qui regarde; pourtant elle ne peut éviter d'y faire face, malgré la peur que ses propres ressources ne soient insuffisantes, la peur que l'avenir n'y change rien. Sa main droite est visible juste devant elle, les doigts repliés d'une façon intrigante; cette main paraît seconder la gravité, le doute irrépressible sensibles sur le visage. Cependant, la main n'est pas fermée, et entre le pouce et l'index replié il ne manquerait qu'un crayon, un stylo... À nouveau encore, les mèches arrondies sur le front portent avec elles l'enfance délicate, mais non fragile : la grâce peut être une immunité, et même une force. Ainsi, les yeux redoutent, mais l'impulsion venue de l'enfance et la main qui peut écrire armeront pour les épreuves. Tel serait le viatique offert à sa fille par une femme chez qui l'observation se fait contemplation, et geste talismanique. 


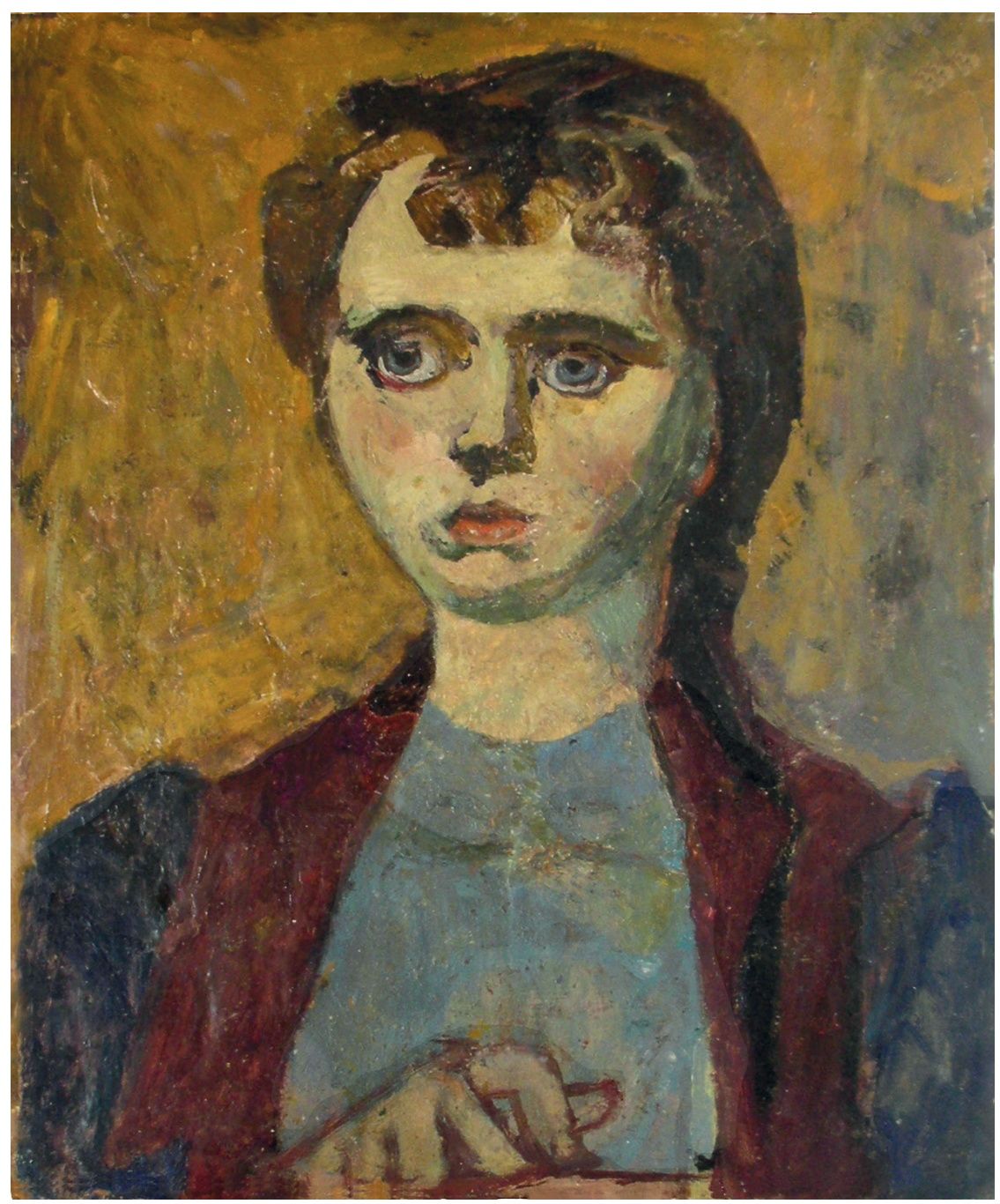

Laura col gilé bordeaux.

\section{Laura con le trecce ${ }^{3}$}

Jeune fille gracieuse, jeune fille prise dans un songe figuré par les paupières décidément baissées. Si délicats sont le cou, l'inclinaison de la tête penchée, l'osmose entre la joue droite et la natte qui la caresse — que l'on pourrait voir là un geste de retrait timide, ou encore une coquetterie

3. Il va s'agir du portrait en plan rapproché, où n'apparaissent que visage et demi-buste, $\mathrm{n}^{\circ} 543 \mathrm{du}$ catalogue. 
trahie par la moue d'une bouche très dessinée, de qui sait charmer en paraissant s'effacer. Cependant, nous ne pouvons nous empêcher de voir ce portrait selon la vocation propitiatoire qui entraîne la peinture de Nella Marchesini. L'enfance de Laura est là, cette mèche rétive au nattage, répondant à l'ombre qui gagne le droit du visage. Mais là encore, l'avant-bras et la main, que l'on ne voit qu'en partie, préfigurent la croissance des forces. Leur coloration plus crue, leur volume suggéré empêchent la réduction de ce portrait à une Grâce juvénile.

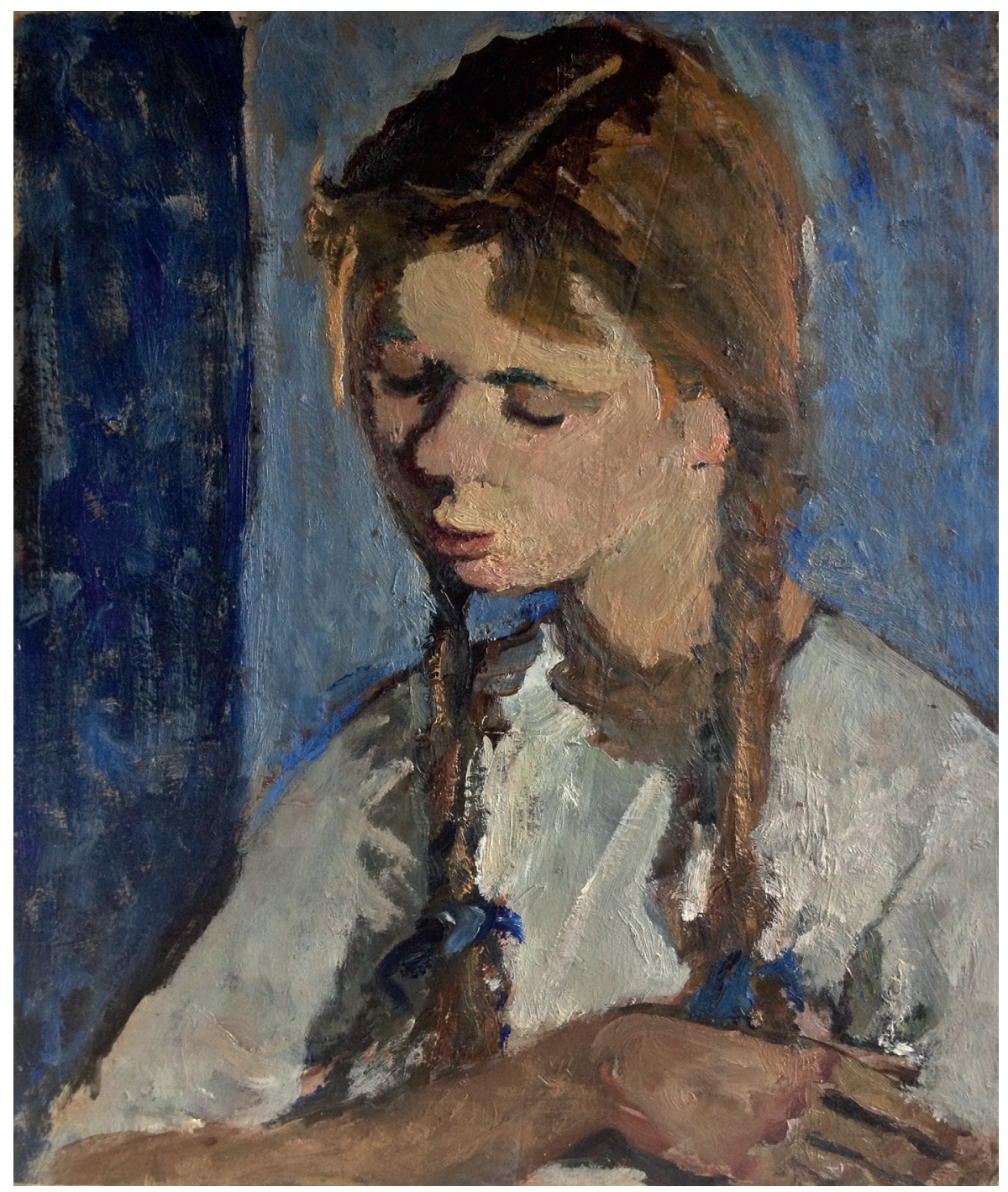

Laura con le trecce. 


\section{Profilo di Laura}

Retenons cette peinture des derniers temps de la vie de Nella Marchesini pour sa légèreté presque bénigne. Ce tableau, sans doute inachevé, fait voir une jeune fille de profil, dont la paupière abaissée paraît enclore une délibération intérieure sans trop de souci, qui n'altère pas le modelé du visage. Le peintre (le mot pittrice manque décidément au français) a marqué sur ce visage un fort contraste lumière/ombre, car le front, l'œil, le nez apparaissent revêtus d'ombre violine. La lèvre supérieure relevée suggère une moue, doute ou coquetterie, qui est mise en lumière juste à la lisière de cette ombre sur le visage. Cependant, une postulation sérieuse prévaut dans ce tableau de la grâce juvénile, à l'image de la coiffure subtile et très ordonnée qui entoure la tête inclinée. Il y a ici un écho de "Laura che fa i compiti», la fillette studieuse rencontrée ci-dessus. Mais le cou, assez fort, est tourné, modelé comme par une main de potière, et c'est une femme qui point dans ce qui est peut-être la dernière vision de Laura donnée par sa mère.

La peinture de Nella a constamment fait deviner la promesse en butte à la menace. Elle a voulu faire paraitre toute ressource, jusqu’à la plus ténue, pour que l'adversité n'ait pas la part trop facile.

\section{Bambine con le trecce}

Maintenant, pour l'œil bleu de Laura, voici le tableau où elle se tient très droite et de face, haussée à la Piero della Francesca, à côté de sa cousine Peppinella, un peu en arrière. Renonçons à évoquer les traits passionnés, dramatiques de ce visage. Voici l'œil bleu qui le résume, comme l'orient de la pierre la plus colorée et la plus transparente à la fois. 


\section{Références des œuvres examinées au fil du texte}

Le texte qui précède n'a pas de prétention d'analyse, ni par conséquent d'analyse raisonnée. Il est seulement porté par l'admiration pour un travail de peinture indéfectible; il serait justifié s'il donnait envie d'aller voir les œuvres de Nella sur le site <www.malvano-marchesini.info> établi avec tant de soin par l'Archivio Ugo Malvano e Nella Marchesini, auquel renvoient les références ci-dessous :

Autoritratto con le figlie: $\mathrm{n}^{\circ} 63 \mathrm{I}$

Laura che fa $i$ compiti : $\mathrm{n}^{\circ} 363$

Laura con la rosa: $\mathrm{n}^{\circ} 362$

Laura con i manichini: $\mathrm{n}^{\circ} 659$

Ritratto della figlia Laura: $\mathrm{n}^{\circ} 368$

Laura sulla poltrone verde: $\mathrm{n}^{\circ} 6 \mathrm{I} 4$

Laura col gilé bordeaux: $\mathrm{n}^{\circ} 6 \mathrm{I} 2$

Laura con le trecce: $\mathrm{n}^{\circ} 543$

Profilo di Laura: $\mathrm{n}^{\circ} 7 \mathrm{I}$

Bambine con le trecce: $\mathrm{n}^{\circ} 396$ 